\section{The Dream Drugstore Chemically Altered States of Consciousness}

by J. Allan Hobson

MIT Press, 320 pp, \$27.95; 2001

\author{
Reviewed by Samuel H. Barondes \\ University of Califomia, San Francisco \\ San Francisco, Califomia, USA
}

On certain nights Allan Hobson, the author of The Dream Drugstore, puts on a cap at bedtime and goes to sleep. Embedded in this nightcap is a device that continuously records the movements of his head. Another device attached to the cap and taped to one eyelid continuously records the movements of his eyeballs. From the data it can be determined when he is awake (both head and eyes move), in deep sleep (neither head nor eyes move) or in the dreaming state, called rapid eye movement (REM) sleep, when the eyes are active but the head is still. Deliberate or spontaneous awakening during REM sleep allows him to relate what he is dreaming about into a tape recorder.

Hobson and others use this approach to examine individual patterns of sleep and to sample the content of dreams throughout the night. They find that the thought processes in dreaming have the same irrational features that are characteristic of the psychotic thinking of people with schizophrenia or severe mood disorders, and of the psychosis induced by drugs like LSD. As Hobson explains in this book, "The main point is that whatever the context-including dreaming-psychosis is psychosis is psychosis. To understand how psychosis can occur naturally, the best approach is to explore the physiology of normal consciousness and learn how the normal psychosis that is dreaming is engendered."

To Hobson, as to many others, a common feature of dreaming, drug-induced psychosis and the psychosis of mental disorders is a change in the comparative activities of serotonin, norepinephrine, dopamine, acetylcholine and other brain chemicals. Called neurotransmitters, these chemicals are released by activated nerve cells, and bind to specific receptors on other nerve cells, thereby transmitting a signal. Hobson reviews the evidence that during REM sleep there is reduced activity of nerve cells that release serotonin and norepinephrine, and a relative preponderance of activity of nerve cells that release acetylcholine. He also reminds us that certain drugs that influence the actions of these neurotransmitters can induce chotic.

None of this is really new, and Hobson has dealt with much of it very effectively in a series of earlier books. For those not familiar with the physiological and neurochemical changes during sleep, he provides a valuable review, although his treatment of other states of consciousness is more limited. The distinguishing feature of The Dream Drugstore is its emphasis on drugs - both the illegal ones, such as LSD, and the legal ones, such as Prozac and related drugs, called selective serotonin reuptake inhibitors (SSRIs). Hobson admits to a "deep-seated bias" against all of them, and believes that "the distinction between the legal and the illegal is now so blurred as to make us realize that we are living in a drug culture on both sides of the law."

Hobson's major complaint about SSRIs is that they increase REM sleep, and that this effect sometimes persists after the drug is discontinued. Although he doesn't explain why this worries him so much, he is concerned that the risks of these drugs may outweigh their benefits. In the final phrase

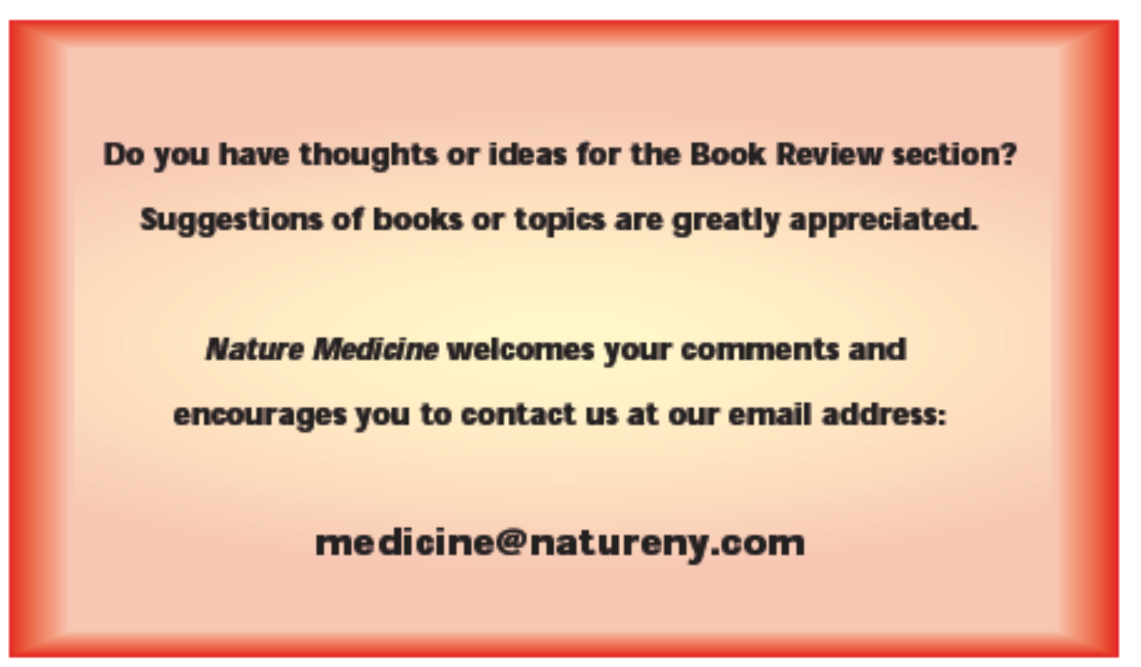

of the book he reminds us that he has offered us ways to "say no to drugs even when they are offered by a friendly psychiatristl ${ }^{\prime}$

Hobson's aversion to SSRIs is hard to reconcile with his personal passion for REM sleep, which these drugs augment. But he doesn't need drugs to experience it. Instead he turns to what he calls his "cerebral pharmacist" - the change in his brain chemistry that naturally occurs during REM sleep. As he puts it, "The drugstore in my brain does the rest."

The problem with Hobson's “deep-seated bias" against psychiatric drugs is that it has allowed him to dismiss them too casually. Although they, like all drugs, are often prescribed inappropriately, there is extensive evidence from placebo-controlled studies that they are effective treatments. Because a warning about their dangers appears to be the main reason he has written this book, he should have more explicitly compared them with their known rewards, which he agrees are substantial. It is fine to send most people to bed with the suggestion that there is pleasure to be derived from the dream drugstore. But for those who are sleepless or otherwise disabled by mental symptoms, it is the real-life who often dispenses relief; and the use of his wares should not be so capriciously discouraged. psychosis, whereas others are anti-psypharmacist in the real-1ife drugstore 